\title{
Transmission of XeCl Excimer Laser Pulses Through Optical Fibers: Dependence on Fiber and Laser Parameters *
}

\author{
G. Hillrichs ${ }^{1}$, M. Dressel ${ }^{1, \star \star}$, H. Hack ${ }^{2}$, R. Kunstmann ${ }^{2}$, and W. Neu ${ }^{1}$ \\ 1 Laser-Laboratorium Göttingen e.V., Im Hassel 21, W-3400 Göttingen, Fed. Rep. Germany \\ 2 Schott Glaswerke, Hattenbergstrasse 10, W-6500 Mainz 1, Fed. Rep. Germany
}

Received 15 October 1991/Accepted 12 December 1991

\begin{abstract}
Measurements of the transmission of $\mathrm{XeCl}$ excimer laser pulses $(308 \mathrm{~nm})$ through optical all-silica fibers are reported. The observed photodegradation effects during long time irradiation are dependent on the laser energy density and the laser repetition rate. Also the influence of the fiber drawing parameters and fiber geometry have been investigated. Absorption spectra of the fibers in the wavelength range from $200 \mathrm{~nm}$ to $400 \mathrm{~nm}$ before and after the irradiation with the excimer laser give some insight in the physical reasons for the changes in transmission properties. A spectral analysis of the fiber fluorescence has been performed.
\end{abstract}

PACS: $78.90,78.40 \mathrm{~F}, 78.20$

Many applications of lasers need the guidance of the laser energy through optical waveguides. Especially most medical applications of $\mathrm{XeCl}$ excimer lasers, e.g. in laser angioplasty $[1,2]$, minimal-invasive surgery [3] and ophthalmology [4] require the use of optical fibers. Most suitable for these purposes are quartz fibers with a core of fused silica and a cladding of doped fused silica. Manufacturing of this kind of fibers became a well established technique. Nevertheless, the interaction of high power UV laser pulses with the fiber material causes specific problems which have been investigated recently by several groups [5-10]. The maximum amount of excimer laser energy which can be transmitted is limited by the damage threshold of the fiber front surface [11, 12]. But already well below this threshold photodegradation effects are observed which lead to a drop of the fiber transmission. It is well known, that UV radiation causes the formation of color centers in the quartz material. Optical fibers are ideal to study these effects, because the optical path can be made long enough to enable the detection of even weak absorption bands. Especially at $308 \mathrm{~nm}$ the effects are usually too small to be observed in experiments with ordinary bulk samples. Getting information on the nature of the absorbing states can help to improve the fiber properties for UV-transmission.

The first scope of this work is to evaluate the influence of several laser and fiber properties on the fiber transmission. Most important laser parameters for the photodegradation

\footnotetext{
* Presented at LASERION '91, June 12-14, 1991, München (Germany)

$\star \star$ Present address: University of Britisch Columbia, Department of Physics, 6244 Agricultural Road, Vancouver B.C. V6T2A6, Canada
}

are the energy density and the repetition rate. Additionally, the influence of the fiber core diameter and fiber drawing conditions has been evaluated. We have also measured fiber absorption spectra in the wavelength range between $200 \mathrm{~nm}$ and $400 \mathrm{~nm}$ before and after laser irradiation and performed a spectral analysis of the fiber fluorescence.

Of practical and of theoretical interest is the fact, that the photodegradation effects are at least partly reversible. The time scale for the recovery effects is measured and discussed together with the temporal development of the fiber absorption spectra after laser irradiation of the fiber has been stopped.

\section{Experimental}

Figure 1 shows the experimental setup for the fiber transmission measurements. The laser (Lambda Physics LPX 210i $\mathrm{CC}$ ) is operated at $308 \mathrm{~nm}$ with a stable resonator. Near the output window the beam dimensions are $8 \mathrm{~mm}$ in vertical and $20 \mathrm{~mm}$ in horizontal direction. The beam divergence is about $1 \mathrm{mrad}$ in the vertical dimension and about $3 \mathrm{mrad}$ in the horizontal. The pulse duration is about $28 \mathrm{~ns}$ FWHM. For variation of the laser energy we use a rotatable dielectric cut-off filter at $308 \mathrm{~nm}$. The attenuation can be adjusted continuously by changing the angle between the laser beam and the filter surface without affecting the temporal and spatial beam profile. Only the central and most homogeneous part of the laser beam is passed through the circular aperture. In order to monitor the energy, which is launched to the fiber, 


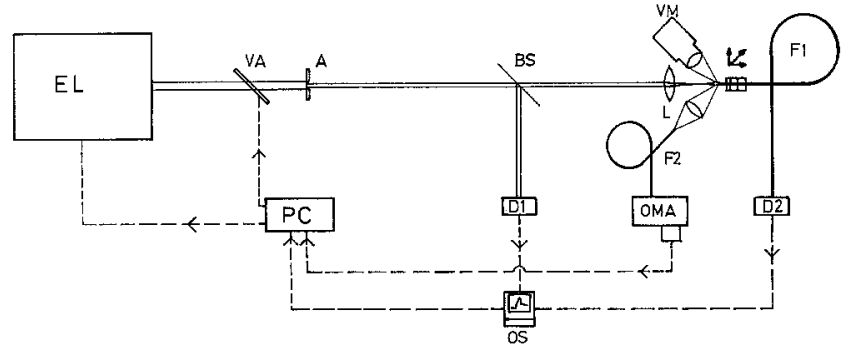

Fig. 1. Experimental setup for the excimer laser measurements. EL: excimer laser. A: aperture, BS: beamsplitter, VM: video microscope, L: lens, F2: fiber for observation of the fluorescence of fiber F1, D1, D2: energy detectors, OMA: optical multichannel analyzer, OS: digital oscilloscope, PC: computer

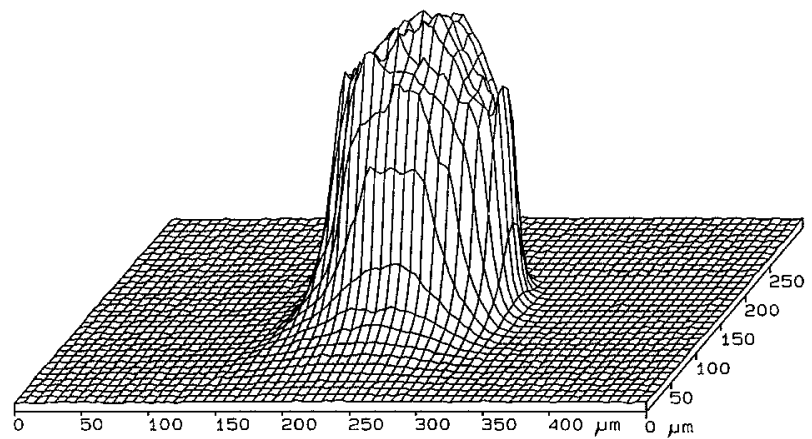

Fig. 2. Profile of the excimer laser beam at the fiber front surface

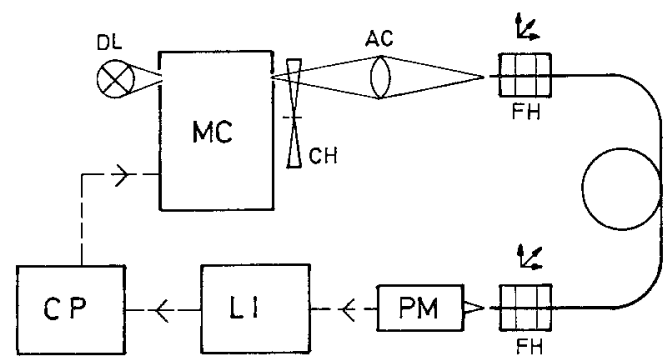

Fig. 3. Experimental setup for measurements of fiber absorption spectra. DL: deuterium lamp, MC: monochromator, $\mathrm{CH}$ : chopper, $\mathrm{AC}$ : UV achromat, FH: fiber holder, PM: photomultiplier, LI: Lock-In amplifier, CP: computer

part of the laser beam is coupled out with a quartz plate and detected by a pyroelectric detector (Laser Precision RjP 735). The laser beam is focused with a biconvex lens with a focal length of $50 \mathrm{~mm}$. Typical numerical apertures of the fibers under test are about 0.2 , while the coupling aperture of the laser beam is less than 0.1. The minimal laser spot size, which can be achieved with this setup has a diameter of about $150 \mu \mathrm{m}$. The spatial beam profile can be checked by using a laser beam profiling system (LLG). A typical profil at the position of the fiber front surface is shown in Fig. 2.

The fiber is fixed in a $v$-groove fiber holder on a $X Y Z$ translation stage. This setup allows adjustment of the fiber relative to the laser spot with an accuracy of about $1 \mu \mathrm{m}$. A microscope $(40 \times)$ in combination with a video camera enables a check of the fiber front surface and allows an easy and reproducable exchange of fibers.

The energy transmitted through the fiber is measured by a second pyroelectric detector (Laser Precision RjP 735). After calibration of this setup the simultaneous reading of the two detector signals by the PC allows the determination of the actual fiber transmission. All long time transmission measurements were performed without interruption of the laser to avoid errors resulting from transmission recovery during irradiation interrupts (cf. Sect. 2.4).

The fluorescence of the fiber near its front surface during irradiation with the excimer laser can be spectrally analysed by an optical multichannel analyzer (OMA). The light emitted from a region of about $5 \mathrm{~mm}$ near the fiber end is guided via a second fiber $(\varnothing 600 \mu \mathrm{m})$ to the OMA system.

For the study of fiber absorption spectra we used the setup shown in Fig. 3. Tunable low intensity UV-radiation $(200 \mathrm{~nm}-400 \mathrm{~nm})$ with a full half width of about $4 \mathrm{~nm}$ is obtained from a deuterium lamp in combination with a scanning monochromator. The exit slit of the monochromator is imaged onto the fiber and the transmitted energy is measured with a photomultiplier. Sensitivity is enhanced by chopping the light and applying the Lock-In technique. When the laser induced changes of the absorption are investigated, first the spectrum of a fiber sample before irradiation is recorded. Then, a certain number of excimer laser pulses is sent through the fiber and the absorption spectrum is recorded again. Comparing both spectra yields the laser induced losses. To reduce adjustment problems as far as possible, the fiber end at which the low intensity radiation from the deuterium lamp is coupled into the fiber is kept in place during all measurements. The excimer laser pulses are then sent through the fiber via the exit side for the deuterium lamp radiation. The readjustment of the fiber in front of the photomultiplier is much less critical.

All fibers used in this work had a core of pure synthetic fused silica and a fluorine doped synthetic fused silica cladding. Typical OH-contents were about $800 \mathrm{ppm}$ to $900 \mathrm{ppm}$. The fibers were prepared for laser transmission by removing the plastic coating from both ends over a length of a few centimeters. Then the front surfaces were prepared by cleaving the fiber by hand (diameters up to $130 \mu \mathrm{m}$ ) or with a fiber cleaver (York FK11). No polishing procedure is applied to avoid any contamination of the surface. Unless otherwise noted, all measurements were performed on $2 \mathrm{~m}$ long samples.

\section{Results}

\subsection{Single Pulse Transmission Properties}

The single pulse attenuation of several quartz fibers has been measured as a function of the laser energy density at the fiber front surface ranging from $2 \mathrm{~J} / \mathrm{cm}^{2}$ to $15 \mathrm{~J} / \mathrm{cm}^{2}$. This corresponds to peak intensities from $70 \mathrm{MW} / \mathrm{cm}^{2}$ to $550 \mathrm{MW} / \mathrm{cm}^{2}$, assuming a pulse duration of $28 \mathrm{~ns}$. For these measurements we used the cut-back method to eliminate the influence of the launching conditions. First, the laser is guided through a sample of $10 \mathrm{~m}$ length and the transmission is recorded for several single pulses. Then, the fiber is cut back from the rear end to a length of $1 \mathrm{~m}$ without changing the launching conditions and the transmission is recorded again. A typical result for three fibers (Schott) is shown in Fig. 4. These fibers were produced with different drawing conditions. The typical value for the single pulse attenuation of the excimer laser radiation at $308 \mathrm{~nm}$ is found to be about $0.15 \mathrm{~dB} / \mathrm{m}$ and is al- 


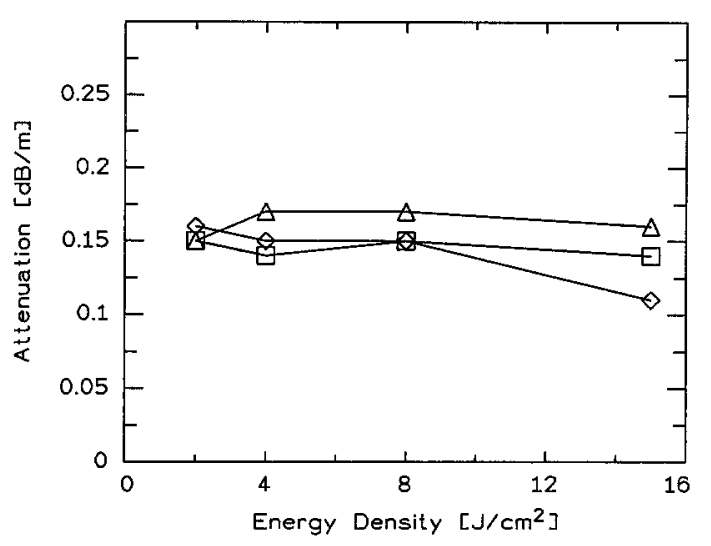

Fig. 4. Attenuation of single $308 \mathrm{~nm}$ excimer laser pulses for different launching energy densities. ( $\square$ Fiber 1; $\triangle$ Fiber 2 ; $\diamond$ Fiber 3)

most independent of the laser energy density. Similar values were obtained with fibers of several different manufacturers. These results agree qualitatively with those obtained in $[8,12]$. It is interesting to note, that within the experimental errors the damping of the low intensity radiation of the deuterium lamp yielded identical values. Unfortunately, we can not give absolute numbers for the lamp intensity at the fiber front surface, but obviously it is orders of magnitude lower compared to the laser intensities. However, it will be shown in the following, that these absorption coefficients given here are only valuable for single pulse conditions or laser repetition rates below about $2 \mathrm{~Hz}$.

\subsection{Long Time Transmission Properties}

A typical long time behaviour of the fiber transmission (core diameter $200 \mu \mathrm{m}$ ) over 100000 laser pulses is shown in Fig. 5. In this example the energy density at the fiber front surface was about $10 \mathrm{~J} / \mathrm{cm}^{2}$ and the pulse repetition rate was $30 \mathrm{~Hz}$. The data have been normalized to the initial single pulse transmission of the fiber at the same energy density, which was $85 \%$ without correction for reflection losses.

The transmission curve shows a strong drop during about 1500 laser pulses. Then a small intermediate increase of fiber transmission is observed, which is followed by a slow and constant decrease of transmission. Qualitatively, this behaviour is very similar for all the fibers which have been investigated. Howewer, some fibers do not show the intermediate increase of transmission.

\subsubsection{Dependence on Energy Density and Pulse Repetition}

Rate. In Fig. 6 the transmission curves are shown for different energy densities at the fiber front surface. From these curves values for the laser induced absorption coefficients after 20000 laser pulses are extracted and shown in Fig. 7. The included absorption shows a linear increase with the laser energy density up to a value near the damage threshold of fused silica.

A typical example of the influence of the laser pulse repetition rate is shown in Fig. 8 (fiber core diameter $200 \mu \mathrm{m}$, energy density $15 \mathrm{~J} / \mathrm{cm}^{2}$ ). Corresponding coefficients for the induced absorption for the various pulse frequencies are plotted in Fig. 9. The solid curve represents a fit of the data with a square root function.

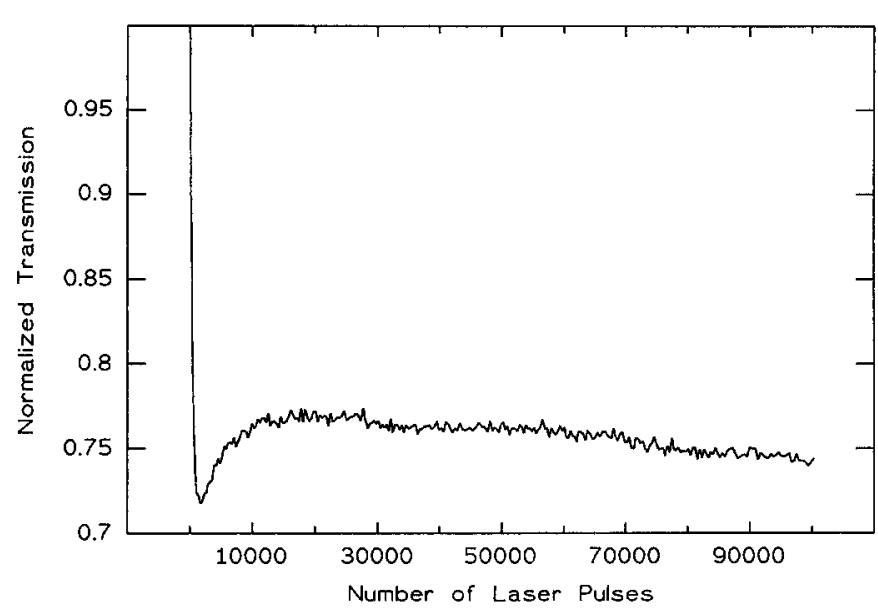

Fig. 5. Long time behaviour of the fiber transmission at a launching energy density of $10 \mathrm{~J} / \mathrm{cm}^{2}$ and a laser repetition rate of $30 \mathrm{~Hz}$

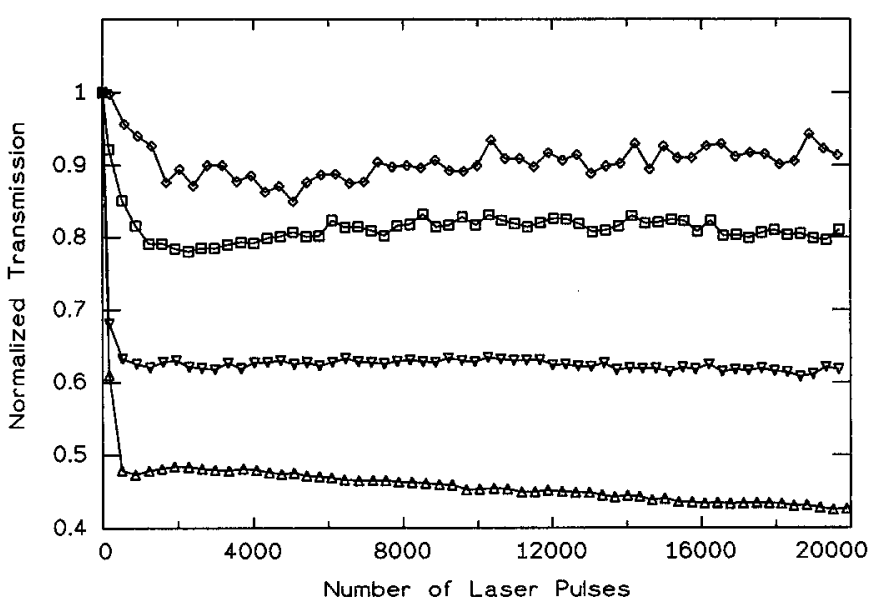

Fig. 6. Fiber transmission at different laser pulse energy densities. Laser pulse repetition rate was $30 \mathrm{~Hz} .\left(\diamond 2 \mathrm{~J} / \mathrm{cm}^{2} ; \square 4 \mathrm{~J} / \mathrm{cm}^{2} ; \nabla 8 \mathrm{~J} / \mathrm{cm}^{2}\right.$; $\triangle 15 \mathrm{~J} / \mathrm{cm}^{2}$ )

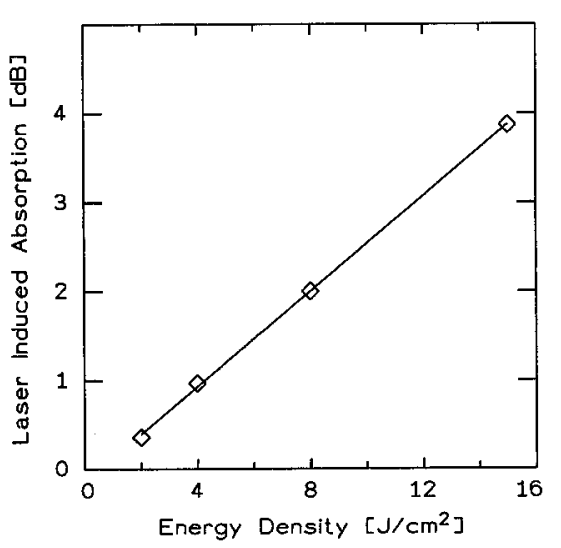

Fig. 7. Laser induced absorption at various launching energy densities. The straight line represents a fit to the data

2.2.2 Dependence on Fiber Parameters. We performed a series of measurements of the photodegradation behaviour with fibers of different core diameters in the range between $100 \mu \mathrm{m}$ and $300 \mu \mathrm{m}$. To confirm identical launching conditions, an aperture $(\varnothing 600 \mu \mathrm{m})$ was illuminated homoge- 


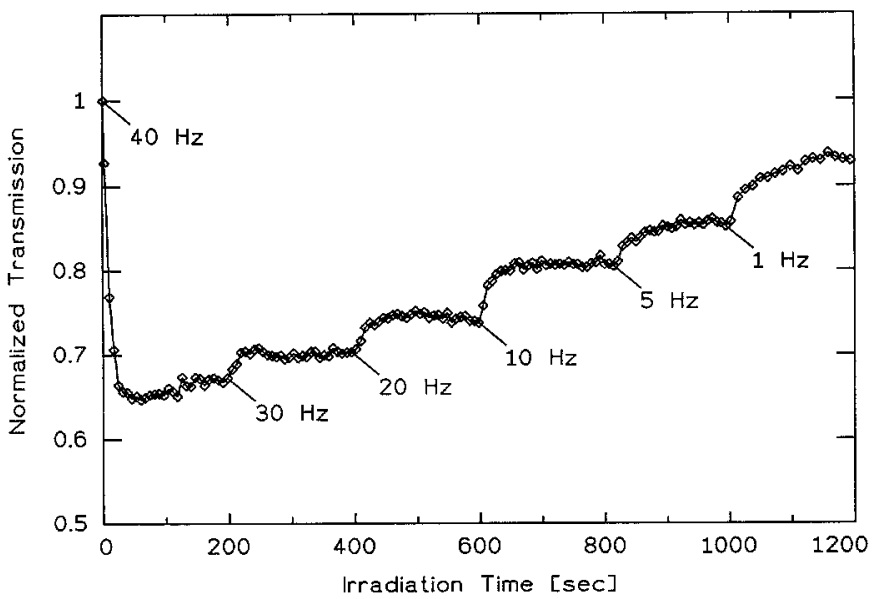

Fig. 8. Fiber transmission at different laser pulse repetition rates. Launching energy density was $15 \mathrm{~J} / \mathrm{cm}^{2}$

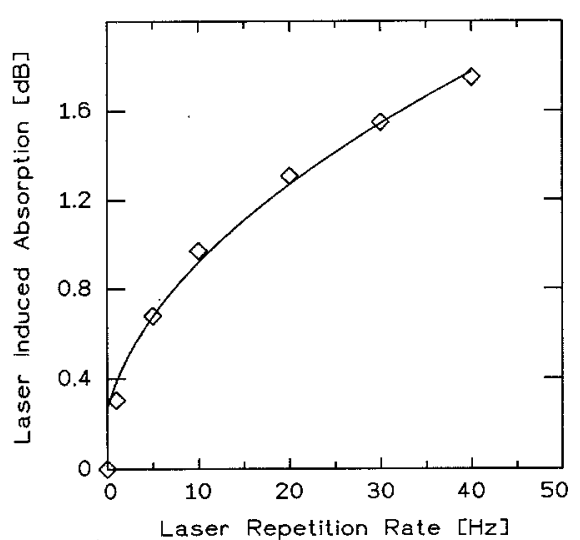

Fig. 9. Laser induced absorption at various laser pulse repetition rates. The solid line represents a square root fit to the experimental data

neously. The fibers were positioned in the center of this aperture under control of the video microscope. It should be noted, that in this setup the laser energy is coupled into the fiber core as well as into the fiber cladding for all fiber diameters used here.

In Table 1 the drop of the normalized transmission after 20000 laser shots is compiled for two sets of fibers from different manufacturers, indicated as $A$ and $B$ in Table 1 . The accuracy of these date is about $\pm 2 \%$. The small differences in the drop of the transmissions and the different behaviour (set $A$ : largest core diameter $\rightarrow$ strongest degradation; set $B$ : largest core diameter $\rightarrow$ smallest degradation) gives rise to the assumption, that the fiber geometry plays a minor role for the photodegradation. Certainly, it is difficult to extract the influence of the fiber dimensions alone, because the drawing conditions during the production of fibers with different dimensions are necessarily different. On the other hand, it will be shown in the following, that the influence of the fiber drawing parameters on the induced absorption is not very strong.

It seems possible, that during the fiber drawing changes in the network of the fiber material are induced, which influence the photodegradation behaviour [13]. In order to study
Table 1. Influence of the fiber core diameter on the photodegradation. Drop of the normalized transmission after 20000 laser pulses with a pulse repetition rate of $30 \mathrm{~Hz}$ and an energy density of $2.6 \mathrm{~J} / \mathrm{cm}^{2}$

\begin{tabular}{lll}
\hline Fiber & $\varnothing[\mu \mathrm{m}]$ & $\begin{array}{l}\text { Transmission } \\
\text { drop }[\%]\end{array}$ \\
\hline $1 A$ & 116 & 11 \\
$2 A$ & 205 & 14 \\
$3 A$ & 300 & 18 \\
$1 B$ & 200 & 15 \\
$2 B$ & 300 & 12 \\
\hline
\end{tabular}

Table 2. Drawing parameters of the fibers, for which the photodegradation is shown in Fig. 10

\begin{tabular}{llll}
\hline Fiber \# & $\begin{array}{l}\text { Drawing } \\
\text { velocity } \\
{[\mathrm{m} / \mathrm{sec}]}\end{array}$ & $\begin{array}{l}\text { Drawing } \\
\text { force } \\
{[\mathrm{cN}]}\end{array}$ & $\begin{array}{l}\text { Drawing } \\
\text { temperature } \\
{\left[{ }^{\circ} \mathrm{C}\right]}\end{array}$ \\
\hline Fiber 1 & 80 & 24 & 2150 \\
Fiber 2 & 5 & 4 & 2150 \\
Fiber 3 & 15 & 5 & 2120 \\
Fiber 4 & 15 & 50 & 1880 \\
\hline
\end{tabular}

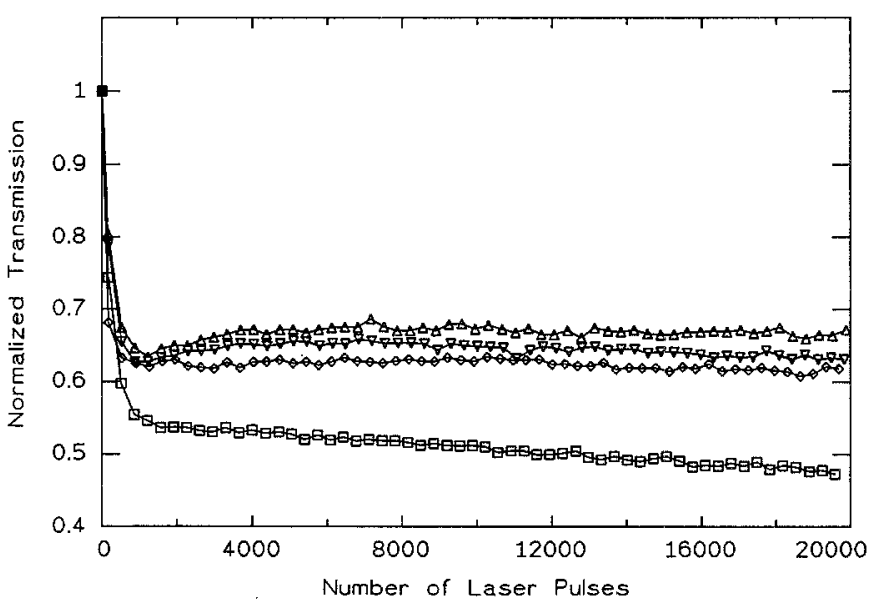

Fig. 10. Transmission of fibers produced with different drawing parameters (see Table 2). ( $\diamond$ Fiber 1; $\square$ Fiber 2; $\nabla$ Fiber $3 ; \Delta$ Fiber 4)

this and to see if a better fiber performance can be achieved by choosing appropriate drawing conditions we performed several measurements on fibers with different production conditions. The results shown in the following were obtained with Schott fibers of similar geometries. Fiber core diameters were about $120 \mu \mathrm{m}$ and the core to cladding ration was 1.1. The drawing parameters are listed in Table 2.

The transmission behaviour of these fibers during 20000 laser pulses is shown in Fig. 10 (laser repetition rate $30 \mathrm{~Hz}$, energy density $15 \mathrm{~J} / \mathrm{cm}^{2}$ ). There is no significant difference in the photodegradation for fibers 1,3 , and 4. Only the fiber with the lowest drawing velocity and drawing force shows a clearly stronger degradation. Qualitatively the same behaviour was found for different launching energy conditions. 


\subsection{Spectral Analysis of the Laser Induced Losses}

The measurements presented up to this point contain information on the laser induced absorption at the laser wavelength itself. Additionally, we performed a spectral analysis of fiber absorption before and after laser irradiation in the spectral range between $200 \mathrm{~nm}$ and $400 \mathrm{~nm}$. In Fig. 11 typical absorption spectra of "fresh" fibers are plotted, taken with the experimental setup shown in Fig. 3. There is a strong

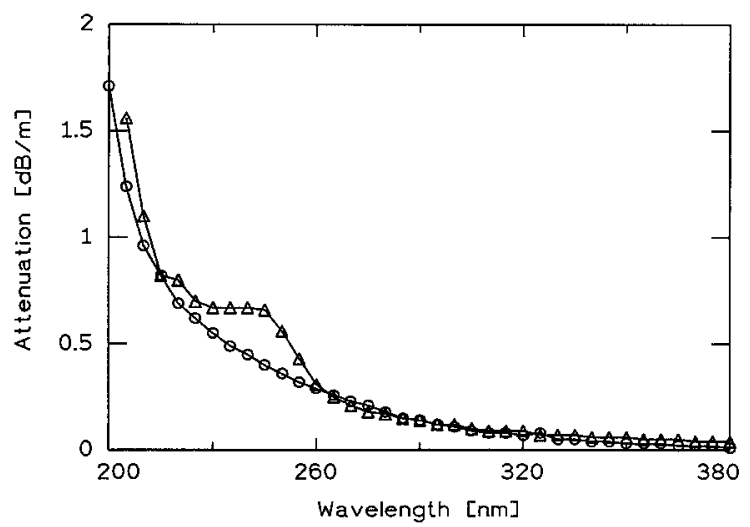

Fig. 11. UV absorption spectra for two different fibers before irradiation with the excimer laser

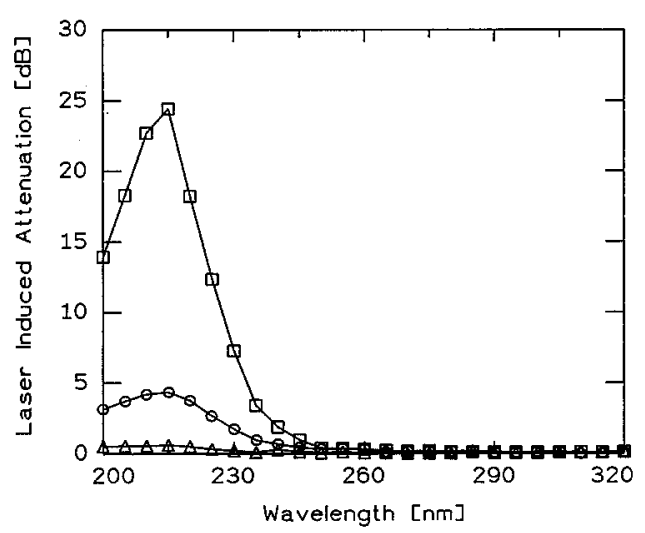

Fig. 12. Spectra of the laser induced fiber absorption after 10,100, and 1000 laser pulses. Launching energy density was $8 \mathrm{~J} / \mathrm{cm}^{2}$, laser pulse repetition rate was $100 \mathrm{~Hz}$. ( $\triangle$ after 10 laser pulses; $\odot$ after 100 laser pulses; $\square$ after 1000 laser pulses) increase of the absorption coefficient with decreasing wavelength. The curve shape is mainly determined by the wavelength dependence of Rayleigh scattering [14]. Some fibers show an additional absorption band around $245 \mathrm{~nm}$.

The additional absorption induced by 10,100 , and 1000 laser pulses of $8 \mathrm{~J} / \mathrm{cm}^{2}$ at a repetition rate of $100 \mathrm{~Hz}$ is shown in Fig. 12. Between the end of the laser irradiation and the recording of these spectra there was a time delay of several minutes. This is important, since recovery effects influence the induced absorption spectrum as will be shown later. It is obvious that already very few laser pulses lead to a strong absorption band around $210 \mathrm{~nm}$, which extends to wavelengths above $300 \mathrm{~nm}$. Figure 13 shows the dependence of the induced absorption in the wing of the $210 \mathrm{~nm}$ band at $245 \mathrm{~nm}$ on the number of laser pulses in a double logarithmic scale.

\subsection{Recovery of Fiber Transmission}

It is reported by several authors, that the drop of fiber transmission by excimer laser radiation is at least partly reversible subsequent to irradiation $[9,10,17]$. The measurements at various repetition rates indicate, that there is a conversion of absorbing centers to non-absorbing states even at a time scale of tenths of seconds. In Fig. 14 the restoration of the

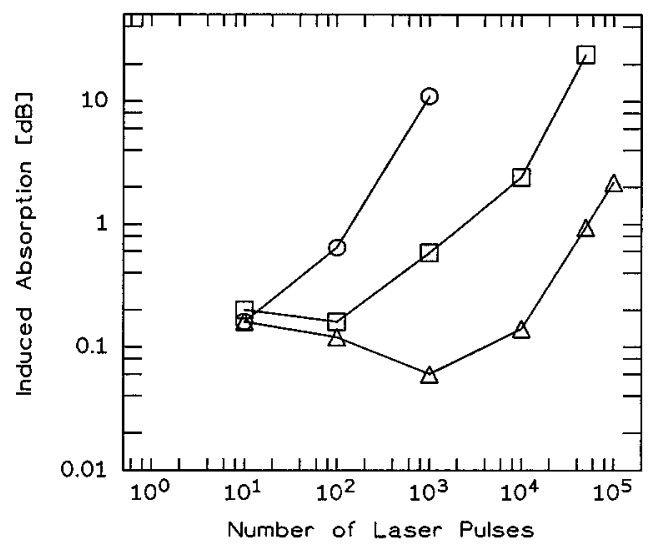

Fig. 13. Laser induced absorption at different wavelengths as a function of the number of laser pulses. Launching energy density was $8 \mathrm{~J} / \mathrm{cm}^{2}$, laser pulse repetition rate was $100 \mathrm{~Hz}$. $(\odot$ at $210 \mathrm{~nm} ; \square$ at $250 \mathrm{~nm}$; $\triangle$ at $310 \mathrm{~nm})$

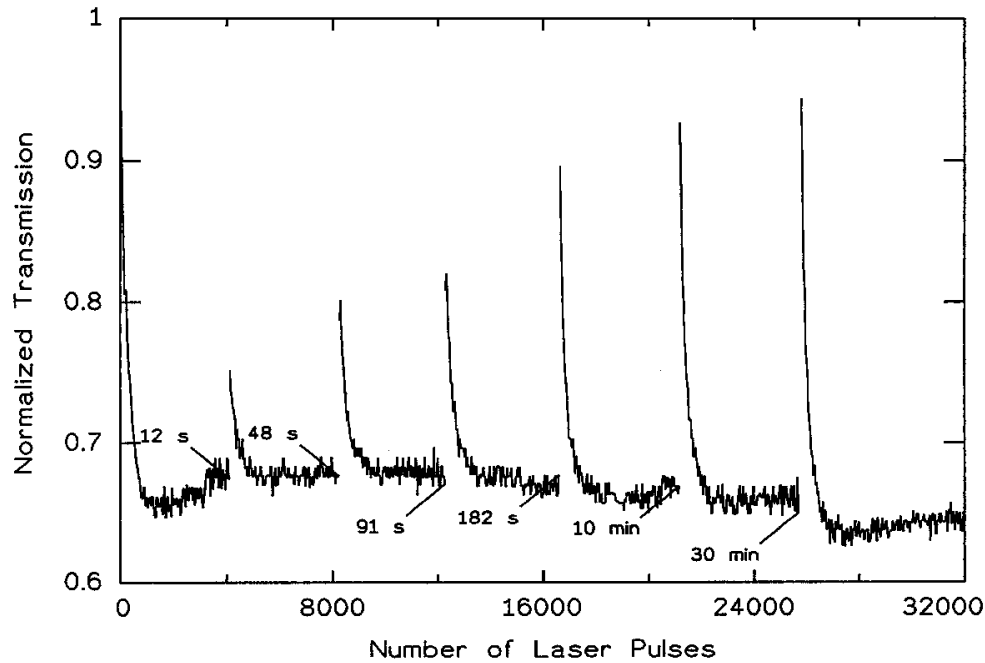

Fig. 14. Transmission of laser pulses through a quartz fiber with interrupts of different lengths, as indicated in the figure. Launching energy density was $15 \mathrm{~J} / \mathrm{cm}^{2}$, laser pulse repetition rate was $30 \mathrm{~Hz}$ 


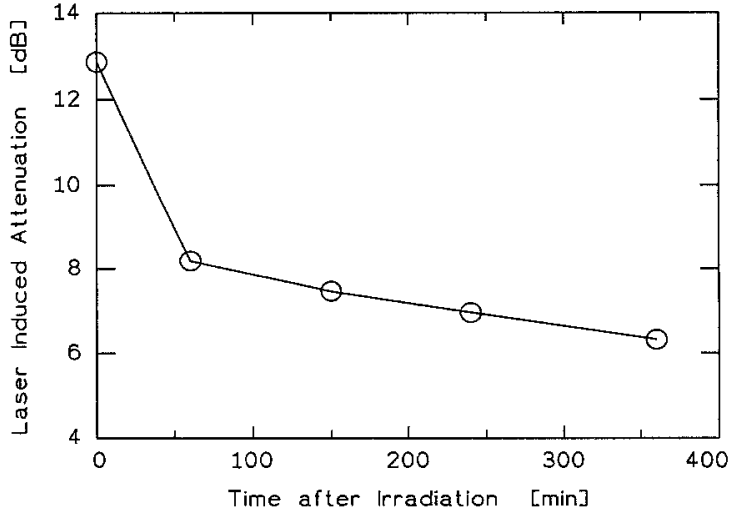

Fig. 15. Annealing of the laser induced absorption at $250 \mathrm{~nm}$ as a function of time after the end of the laser irradiation

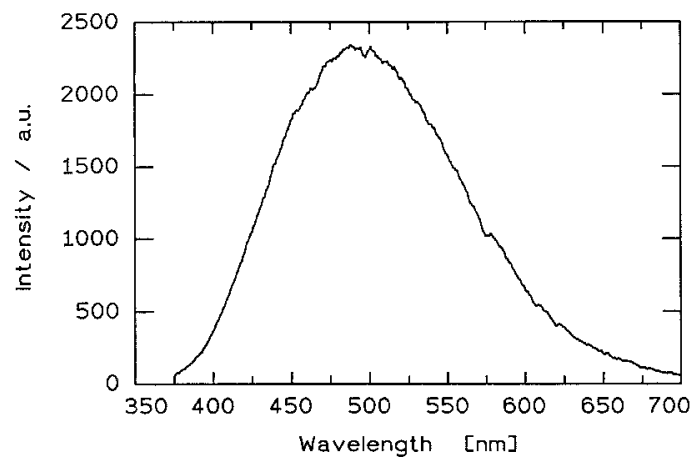

Fig. 16. Spectrum of the laser induced fiber fluorescence

transmission during irradiation interrupts of different temporal length are shown. After a few minutes the initial transmission of the fiber is reached again. Switching on the laser again leads to a new drop of the fiber transmission to the same level as prior to the irradiation interrupt.

The temporal behaviour of the laser induced absorption in the wing of the $210 \mathrm{~nm}$ band at $250 \mathrm{~nm}$ is shown in Fig. 15 . Partly vanishing of the induced absorption is found. However, even $24 \mathrm{~h}$ after the end of fiber irradiation there is still a remaining absorption.

\subsection{Spectral Analysis of the Fiber Fluorescence}

To get more information about the physigal reasons for the decrease of transmission we have performed a spectral analysis of the fiber fluorescence. Near the fiber front surface the fiber fluoresces during the irradiation with the excimer laser. A typical result is shown in Fig. 16. The spectrum shown was accumulated over 20 laser pulses. We have found an unstructured and broad fluorescence band arond $450 \mathrm{~nm}$, which, qualitatively, is very similar for many different fibers.

\section{Discussion}

Transmission of single $\mathrm{XeCl}$ excimer laser pulses through optical fibers is nearly independent of laser energy density up to the damage threshold of the fiber front surface. This result is in agreement with similar investigations of Brimacombe et al. [8]. The typical attenuation coefficient of about $0.15 \mathrm{~dB} / \mathrm{m}$ approaches the theoretical limit [14]. For single pulses or repetition rates of less than about $2 \mathrm{~Hz}$ the limiting factor for transmission of $\mathrm{XeCl}$ excimer laser pulses is the surface damage threshold of fused silica. Values for this threshold have not been determined here, but previous investigations yielded a typical value of about $20 \mathrm{~J} / \mathrm{cm}^{2}$, however, this value depends on the definition of the damage $[11,12]$. Irradiation of the fiber cladding reduces this value considerably.

For applications, which need a long time transmission at higher laser pulse frequencies, the strong drop of transmission after the first laser pulses has to be taken into account. We found an increase of the laser induced absorption proportional to the square root of the laser repetition rate $f$. In contrast to the single pulse transmission the multiple pulse transmission is dependent on the laser energy density $F$. The absorption coefficient $d$ increases linearly with the energy:

$d \sim F \times f^{1 / 2}$.

A similar relation for the laser induced absorption was derived by Konov et al. [9].

The independence of the transmission drop from the fiber diameter indicates, that the photodegradation is a property of the fused silica of the fiber core and is independent from the fluorine doped fused silica of the cladding and especially from the interface between core and cladding.

The typical transmission curves (see e.g. Fig. 5) indicate, that absorbing states are produced by the first laser pulses. The following pulses are attenuated by these absorption centers. Under room temperature conditions the absorbing states are not stable as shown by the increase of the transmission during irradiation interrupts. After a few minutes the initial transmission is recovered again (see Fig. 14). The amount of the initial drop of transmission during a few thousand laser pulses is defined by an equilibrium between production of absorption centers by laser irradiation and by the rate of restoration of these defects. For the further slow decrease of transmission after a larger number of pulses a second process is responsible, which is based on two-photon absorption (see [19] and references therein).

The absorption spectra taken after irradiation of the fiber with the excimer laser show a strong absorption band around $210 \mathrm{~nm}$, which has been identified as a result of the $E^{\prime}$-color centers [19]. It is known, that this band is also induced by $193 \mathrm{~nm}$ and $248 \mathrm{~nm}$ excimer laser radiation [15] and also by broad band UV light emitted by a deuterium lamp [16]. Very recently it was found by Fabian et al. [16], that the intensity of the UV-induced absorption band at $210 \mathrm{~nm}$ can be influenced by treating the fiber preform or the fiber itself with hydrogen under high pressure or high temperature conditions.

The observation of an unstructured wing of the $210 \mathrm{~nm}$ band in the induced absorption spectra in Fig. 12 up to more than $300 \mathrm{~nm}$ leads to the assumption, that decreasing transmission of $308 \mathrm{~nm}$ radiation is influenced by generation of $E^{\prime}$-centers. Several authors suppose, that the transmission drop at $308 \mathrm{~nm}$ is connected with another type of color centers, the non-bridging oxygen hole centers $[9,10]$. However, 
the appearance of this absorption center normally coincides with the appearance of red fluorescence light around $650 \mathrm{~nm}$ which had been observed during transmission of $248 \mathrm{~nm}$ excimer light through fused silica fibers $[10,17]$. Our spectral analysis of the fluorescence of several different fibers during irradiation with $308 \mathrm{~nm}$ laser radiation have shown only a broad fluorescence band around $450 \mathrm{~nm}$. This fluorescence is connected with a $245 \mathrm{~nm}$ absorption band (see Fig. 11) caused by an oxygen deficit of the material [17]. Contrary to our experiments Müller et al. [18] observed the additional fluorescence band around $650 \mathrm{~nm}$ during irradiation of fibers with $308 \mathrm{~nm}$ radiation. Therefore, we suppose, that non-bridging oxygen hole centers affect the transmission of $308 \mathrm{~nm}$ laser photons, but this process strongly depends on individual fiber properties.

As already mentioned, it was observed by Fabian et al. [16], that broad band UV radiation causes an increase of the $210 \mathrm{~nm}$ absorption band. They also found a small decrease of absorption for wavelengths longer than $250 \mathrm{~nm}$. This has been explained by a reaction of hydrogen molecules present in the quartz material with defects in the glass structure, converting these defects into non-absorbing states. The activation energy for this process is supplied by the UV-radiation. A similar behaviour under $308 \mathrm{~nm}$ laser radiation may cause the local minimum in the transmission curve after a few thousand laser pulses, which has been observed for several fibers (see Fig. 5). Different dependencies on the laser doses of the $210 \mathrm{~nm}$ band and of the hydrogen reaction may lead to the structure in the transmission curve for $308 \mathrm{~nm}$ laser radiation. However, this assumption should be confirmed in further experiments.

Leclerc etal. [19] have shown that after irradiation of blocks of OH-rich synthetic fused silica with $248 \mathrm{~nm}$ laser radiation there is also an almost complete recovery of the initial transmission during an irradiation interrupt. Switching on the laser again leads immediately to a rebuilding of the absorption bands. Based on these observations the relaxation of the laser induced $E^{\prime}$-centers not to the original state but to a new non-absorbing state has been proposed. This state can be easily converted into an absorbing state by a second laser irradiation. Our experiments at $308 \mathrm{~nm}$ have shown a very similar rate of transmission drop of a "fresh" fiber and a previously irradiated fiber (cf. Fig. 14). So this can be an indication for a qualitative difference in the fiber behaviour under $308 \mathrm{~nm}$ and $248 \mathrm{~nm}$ irradiation. On the other hand, the dependence of the $\mathrm{KrF}$ laser induced attenuation coefficient at $210 \mathrm{~nm}$ on the repetition rate has also been observed [19]. This dependence is very similar to what we found for $\mathrm{XeCl}$ laser induced absorption at $308 \mathrm{~nm}$ (Fig. 9). This is a further indication that the $210 \mathrm{~nm}$ band strongly influences the transmission drop of $308 \mathrm{~nm}$ as well as of $248 \mathrm{~nm}$ laser radiation.

The temporal behaviour of the decrease of the laser induced $210 \mathrm{~nm}$ absorption band (see Fig. 15) shows that after laser irradiation the fiber absorption spectrum is changed for several hours. This is also of practical importance if the fiber is used simultaneously for laser transmission and for guiding laser induced fluorescence back from the laser treated area [20]. Our data do not allow the determination of the recovery behaviour within the first minutes after the irradiation. This requires an experimental setup similar to that devel- oped by Leclerc et al. [17] used to study the fiber absorption after irradiation with $248 \mathrm{~nm}$ radiation. They found a strong decrease of the magnitude of the $210 \mathrm{~nm}$ absorption during the first seconds after irradiation for $\mathrm{OH}$-rich fibers.

The experiments with fibers produced with different drawing parameters have shown that the fiber performance for UV transmission is independent of these parameters over a wide range. Only the fiber with the lowest drawing velocity and drawing force has shown a higher photodegradation. It seems possible that at low drawing velocities the time spent in the hot region of the drawing apparatus it too long, so that there is more time for impurities to diffuse into the fiber and worsening the fiber properties.

\section{Conclusions}

The most important parameters for long time transmission of $\mathrm{XeCl}$ excimer laser radiation are the laser energy density and the laser repetition rate. An increase of the energy and of the repetition rate of the laser lead to a decrease of the fiber transmission. After several thousand laser pulses the transmission is nearly constant. Interruption of laser irradiation leads to a total restoration of the transmission, however, switching on the laser again causes a new drop of transmission.

The photodegradation is independent of the fiber core diameter and of the fiber drawing parameters, as long as the drawing velocity and force are not to low.

The study of the laser induced absorption spectra and the fiber fluorescence lead to the assumption that the wing of the $210 \mathrm{~nm}$ absorption band is mainly responsible for the decrease of the laser transmission. However, all processes which contribute to the drop of transmission have further to be investigated to allow an improvement of the fiber performance for transmission of UV excimer laser radiation.

Acknowledgements. Parts of this work were supported by the Bundesministerium für Forschung und Technologie, grant 13N5669. Many ideas have been developed together with $\mathrm{H}$. Gerhardt. A. Spata assisted us in all technical aspects and the performance of most of the experiments. We thank them for their cooperation.

\section{References}

1. D.L. Singleton, G. Paraskevopoulos, R.S. Taylor, L.A.J. Higginson: IEEE J. QE-23. 1772 (1987)

2. K.R. Karsch, K.K. Haase, M. Mauser, W. Voelker: Am. J. Card. 64, 1253 (1989)

3. R. Jahn, M. Dressel, H. Fabian, K.F. Klein, H.U. Langendorff, W. Neu, K.H. Jungbluth: Laser Med. Surg. 6 (1990)

4. M. Vogel, G. Scheurer, W. Neu, M. Dressel, H. Gerhardt: Klin. Monatsbl. Augenheilk. 196, 250 (1990)

5. R. Pini, R. Salimbeni, M. Vannini: Appl. Opt. 26. 4185 (1987)

6. C. Whitehurst, M.R. Dickinson, T.A. King: J. Mod. Opt. 35, 371 (1988)

7. R.S. Taylor, K.E. Leopold, R.K. Brimacombe, S. Mihailov: Appl Opt. 27, 3124 (1988)

8. R.K. Brimacombe, R.S. Taylor, K.E. Leopold: J. Appl. Phys. 66, 4035 (1989) 
9. V.I. Konov, V.P. Pashinin, A.S. Silenok: SPIE 1201, 247 (1990)

10. V.G. Artjushenko, V.I. Konov, V.P. Pashinin, S.S. Silenok, L.M. Blinov, A.M. Solomatin, I.P. Shilov, V.V. Volodko, G. Müller, B. Schaldach, R. Ulrich, W. Neuberger: SPIE Proc., Los Angeles (1991), to be published

11. R.S. Taylor, K.E. Leopold, S. Mihailov, R.K. Brimacombe: Opt. Commun. 63, 26 (1987)

12. M. Dressel, W. Neu, H. Gerhardt: Laser und Optoelektronik 22, $76(1990)$

13. P. Kaiser: J. Opt. Soc. Am. 64, 475 (1974)

14. Lines, MacChesney, Lyons, Bruce, Miller, Nassau: J. Non-Cryst. Solids 107, 251 (1989)
15. T.E. Tsai, D.L. Griscom, E.J. Friebele: Phys. Rev. Lett. 61, 444 (1988)

16. H. Fabian, U. Grzesik, K.-H. Wörner: SPIE 1513, 168 (1991)

17. N. Leclerc, C. Pfleiderer, H. Hitzler, S. Thomas, R. Takke, W. Englisch, J. Wolfrum, K.-O. Greulich: SPIE 1327, 60 (1990)

18. G. Müller, H. Kar, K. Dörschel, H. Ringelhan: SPIE 906, 231 (1988)

19. N. Leclerc, C. Pfleiderer, H. Hitzler, J. Wolfrum, K.-O. Greulich, S. Thomas, H. Fabian, R. Takke, W. Englisch: Opt. Lett. 16, 940 (1991)

20. W. Neu, K.K. Haase, C. Tischler, R. Nyga, K.R. Karsch : SPIE 1425, 28 (1991) 